\title{
The Deletion of TRPC6 Channels Perturbs Iron and Zinc Homeostasis and Pregnancy Outcome in Mice
}

\author{
Jessy Hasna ${ }^{a, b, c}$ Roland Abi Nahed ${ }^{b, c, d} \quad$ Frédéric Sergent ${ }^{b, c, d} \quad$ Nadia Alfaidy ${ }^{b, c, d}$ \\ Alexandre Bouron ${ }^{\mathrm{a}, \mathrm{b}, \mathrm{c}}$ \\ aUniversité Grenoble Alpes, CNRS, CEA, BIG-LCBM, Grenoble, France, 'Commissariat à l'Energie \\ Atomique et aux Energies Alternatives (CEA), Biosciences and Biotechnology Institute of Grenoble, \\ Grenoble, France, 'University Grenoble-Alpes, Grenoble, France, dInstitut National de la Santé et de la \\ Recherche Médicale, Grenoble, France
}

\section{Key Words}

TRPC $・$ Ion channels $\bullet$ Metals $\bullet$ Brain $\bullet$ Placenta

\begin{abstract}
Background/Aims: Transient receptor potential canonical 6 (TRPC6) protein is a nonselective cation channel permitting the uptake of essential elements such as iron $(\mathrm{Fe})$ and zinc (Zn). TRPC6 is found throughout the body with high expression levels in the placenta. However, its role in this organ is still to be determined. To further advance our understanding of the physiological relevance of TRPC6, we have studied the placental histology, pregnancy outcome and the Fe and Zn status of organs (placenta, brain, kidney, liver and lung) collected from TRPC6 deficient (TRPC6 ${ }^{-/}$) mice and sex and age-matched C57BI6/J and B6129SF2/J mice. Methods: Metal content was quantified by inductively coupled plasma-atomic emission spectrometry (ICP-AES). Quantitative reverse transcriptase PCR (qRT-PCR) and Western Blottings (WB) were performed to analyze the expression of placental markers and TRPC6. Results: Our data show that TRPC6 ${ }^{-/}$mice displayed reduced litter sizes, structural changes of the placenta, along with altered mRNA levels of CD31 and Gcm1, two markers of placental development. Furthermore, immunoblots revealed elevated amounts of TRPC 6 proteins in placentas from women diagnosed with preeclampsia, a common gestational disease. When compared to C57BI6/J and B6129SF2/J, TRPC6 ${ }^{--}$mice had elevated $\mathrm{Zn}$ levels in placenta, liver and kidney during embryonic development and postnatally, but not at adulthood. High amounts of Fe were found in the adult brain and liver of TRPC6 ${ }^{-1}$ mice. The lung was however not affected by the deletion of TRPC6, indicating that this mouse strain developed organ and age-dependent perturbations in their $\mathrm{Zn}$ and Fe status. Conclusion: This work indicates that TRPC6 exerts critical pathophysiological functions in placenta, and provides further evidence for a role of this channel in the homeostasis of cations like $\mathrm{Zn}$ and Fe.
\end{abstract}




\section{Cellular Physiology Cell Physiol Biochem 2019;52:455-467 \\ \begin{tabular}{ll|l} 
and Biochemistry & $\begin{array}{l}\text { DOl: } 10.33594 / 000000033 \\
\text { Published online: } 15 \text { March } 2019\end{array}$ & $\begin{array}{l}\text { C } 2019 \text { The Author(s). Published by } \\
\text { Cell Physiol Biochem Press GmbH\&Co. KG }\end{array}$
\end{tabular} \\ Hasna et al.: TRPC6 in Placenta and Mineral Homeostasis}

\section{Introduction}

The protein transient receptor potential canonical (TRPC) 6 is a member of the TRPC family. Although it can be found intracellularly, TRPC6 is merely regarded to function as a plasma membrane cation channel [1]. Diverse stimuli can enhance its activity. For instance, TRPC6 opens in response to the stimulation of some G-protein coupled receptors, after a direct application of diacylglycerol (or diacylglycerol analogues) [2], or in response to hyperforin [3], a bioactive molecule extracted from the medicinal herb Hypericum perforatum. Whatever the activation mode, the opening of TRPC6 channels triggers an influx of sodium (Na) and calcium (Ca) ions [4]. In addition, electrophysiological and imaging experiments conducted on HEK cells, the neuronal cell line PC12 and cortical neurons in primary cultures proposed that TRPC6 could also function as an uptake pathway for essential metals like iron (Fe) [5, 6] and zinc (Zn) [7, 8]. Furthermore, the stable over-expression of TRPC6 in HEK-293 cells causes an intracellular $\mathrm{Zn}$ overload, a feature not found in HEK cells overexpressing its close relative TRPC3 [7]. These data suggested that the non-selective Ca-conducting cation channel TRPC6 [4] could play a role in the mineral status of cells. Since the cloning of TRPC6 from murine brain tissue [9] many groups have provided significant insight into its expression and functions $[1,10]$. For instance, TRPC6 protein displays a large distribution profile, detected in many organs and tissues like muscles, brain and skin with high expression levels in placenta and lung $[2,11]$. Although early and late term placenta express TRPC6 [2,11-13], its role in placental development and pregnancy outcome is currently unknown.

This study was initiated to gain further knowledge on the physiological relevance ofTRPC6 in the placenta. We compared the placentation and pregnancy outcome of TRPC6 deficient (TRPC6 ${ }^{-/}$) mice with sex and age-matched C57Bl6/J and B6129SF2/J mice. The results obtained showed that the loss of TRPC6 was associated with histological modifications of the placenta, altered mRNA levels of some placental markers (CD31 and Gcm1), together with a reduced litter size. Measurements of $\mathrm{Zn}$ and Fe contents with inductively coupled plasmaatomic emission spectrometry (ICP-AES) revealed that these changes were accompanied by a Zn enrichment in embryonic and neonatal tissues notably the placenta, lung and kidney, whereas liver and brain from adult mice displayed elevated amounts of Fe. The metal content of the lung was however unaffected by the absence of TRPC6. This indicated that the deletion of the TRPC6 gene generated organ and age-dependent perturbations in $\mathrm{Zn}$ and Fe status. To further document the physiological relevance of TRPC6 channel in pregnancy, we compared the amounts of TRPC6 proteins in preeclampsia (PE), one of the most threatening human pregnancy pathology. This disease affects $5-6 \%$ of all pregnancies and is associated with a strong deregulation of the mineral status of the placenta [14]. Immunoblots showed elevated TRPC6 protein levels in PE placentas compared to control placentas, suggesting a potential compensatory role of this protein. Overall, these findings indicate that TRPC6 exerts pathophysiological relevant functions in placental development and pregnancy. They also reinforce the emerging view of TRPC6 as an actor of the homeostasis of essentials elements like Fe [5] and Zn [7, 8].

\section{Materials and Methods}

Animals and tissue collection

C57Bl6/J (stock \#000664), B6129SF2/J (stock \#101045) mice and TRPC6 knockout mice (TRPC6\%) (\#101045) [15] were obtained from the Jackson Laboratory (USA). Animals were housed in the same room under a $12 \mathrm{hr}$ light - $12 \mathrm{hr}$ dark cycle with ad libitum access to food and water. They were exposed to an enriched environment in agreement with the Animal Welfare Committee of the CEA Grenoble. All the cages were located on the same rack and they were changed at the same frequency (once a week), the same day. Mice were sacrificed using cervical dislocation. Fresh tissues (placenta, brain, kidney, liver and lung) were rapidly isolated and weighted. The experimental protocol was approved by the ethical committee of the 


\section{Cellular Physiology Cell Physiol Biochem 2019;52:455-467

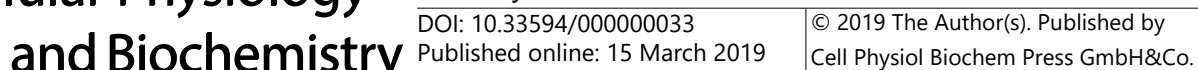 \\ Hasna et al.: TRPC6 in Placenta and Mineral Homeostasis}

CEA's Life Sciences Division (CETEA) (A14-006). Since the presence of a vaginal plug is not a reliable marker of pregnancy, animals were crossed at night once a week to ease the dating of the embryos. Males were removed the morning before 9 a.m. and this day was counted as E0.

\section{Human placentas}

Procedures were as already described [16]. Collection and processing of human placentas were approved by the district and local hospital ethical committees, and collection was performed according to the Mount Sinai Hospital and University of Toronto code of practice. Informed patient consent was obtained in all cases. The preeclamptic group was selected to represent classic preeclampsia (PE) according to both clinical and pathological criteria [17]. Controls patients were primiparous but did not show clinical or pathological signs of PE or any other placental disease [16]. Preeclamptic and gestational age-matched control placentas were collected between 24 and 39 weeks of gestation (wg) (Table 1). PE was defined as a diastolic arterial blood pressure greater than $90 \mathrm{~mm} \mathrm{Hg}$ and a systolic blood pressure greater than 140 $\mathrm{mm} \mathrm{Hg}$, associated with proteinuria (more than $300 \mathrm{mg} / 24 \mathrm{~h}$ ). Blood pressure was taken at admission and at the inclusion day, after at least half an hour of bed rest. Placentas were obtained within 20 min of delivery. Placental tissue samples were excised from random areas of the placental cotyledons, excluding the peripheral margin and infarcted areas. Samples from each placenta were snap frozen and stored at $-80^{\circ} \mathrm{C}$ for protein extraction.

\section{Analysis of the metal content in organs}

The iron (Fe) and zinc (Zn) content was determined by inductively coupled plasma atomic emission spectroscopy (ICP-AES, ICPE-9000 from Shimadzu Scientific Instruments). Experiments were conducted as follows: organs were rapidly collected and weighted before being dried at $60^{\circ} \mathrm{C}$ for $24 \mathrm{~h}$ on a DigiPREP Block digestion system. Dried tissues were mineralized overnight in $65 \%$ nitric acid. Mineralized samples were then diluted with purified water to attain a final concentration of $10 \%$ nitric acid. A multi-element standard (Sigma-Aldrich) was used for calibration. Ytterbium (Sigma-Aldrich) was added at $100 \mu \mathrm{g} / \mathrm{L}$ to all solutions (samples and standard) as an internal control. The data obtained were analyzed with the ICPE9000 analysis software by selecting wavelengths displaying minimal interference with other elements. Each sample was analyzed in triplicate. Only values with a relative standard deviation lower than $2 \mu \mathrm{g} / \mathrm{L}$ were retained. Results are presented in ng of metal/mg of dried organ.

\section{Retro-transcription and quantitative real-time PCR (RT-qPCR)}

Total RNA was isolated from murine placentas at embryonic age E13 using the Nucleospin® RNA Kit (Macherey-Nagel) according to the manufacturer's instructions. RNA was quantified with a NanoDrop ND1000 spectrophotometer by means of the ND1000 software (Labtech). $0.5 \mu \mathrm{g}$ of total RNA was retrotranscribed into cDNA using the Affinity Script cDNA Synthesis kit (Agilent Technologies) and the $\mathrm{C}_{1000^{\mathrm{TM}}}$ Thermal Cycler (Biorad). Gene expression levels were determined by qPCR using Brilliant II SyBR Green qPCR Mastermix (Agilent) and the CFX 96 ${ }^{\mathrm{TM}}$ Real-Time system (Bio-Rad). Data were analyzed with the BioRad CFX Manager software (version 2.0). All qPCR primers (Table 2) were designed with NCBI Primer3/ BLAST (Basic Local Alignment Search Tool) and purchased from Eurofins Genomics. They were dissolved in water and used at a final concentration of $5 \mu \mathrm{M}$. GAPDH, and RPL13a were used as reference genes. Experiments were conducted in triplicate on RNAs extracted from 3 independent biological samples.

Table 1. Clinical characteristics of normal and preeclamptic patients. Differences between the two groups were measured with a Student's t-test. n.s. non significant

\begin{tabular}{lccc}
\hline Parameter & Normal pregnancies $(\mathrm{n}=10)$ & Preeclamptic pregnancies(n=9) & $\mathrm{p}$ \\
\hline Maternal Age (y) & $31.1[21-39]$ & $32.2[24-40]$ & $\mathrm{ns}$ \\
Gestational age at sampling (in weeks) & $30.6[22-35]$ & $27.7[25-32]$ & $\mathrm{ns}$ \\
Parity & $0.4[0-2]$ & $0.4[0-2]$ & $\mathrm{ns}$ \\
Fetal weight (g) & $2,147[1,110-4,905]$ & $1,554[1,110-1,955]$ & $\mathrm{ns}$ \\
\hline
\end{tabular}




\section{Cellular Physiology Cell Physiol Biochem 2019;52:455-467 \begin{tabular}{ll|l|l}
\cline { 2 - 2 } DOI: 10.33594/000000033 & () 2019 The Author(s). Published by
\end{tabular} and Biochemistry Published online: 15 March 2019 Cell Physiol Biochem Press GmbH\&Co. KG \\ Hasna et al.: TRPC6 in Placenta and Mineral Homeostasis}

\section{Protein preparation}

Placentas were homogenized with a mixer (Polytron 1200 mixer, Bioblock Scientific, France) in RIPA (Tris-HCl 2, $42 \mathrm{~g}, \mathrm{NaCl} 8,77$ $\mathrm{g}$, sodium deoxycholate $5 \mathrm{~g}$, sodium dodecyl sulphate (SDS) $1 \mathrm{~g}$, Triton X-100 $10 \mathrm{ml}, \mathrm{pH}$ 8) containing a cocktail protease inhibitor (Sigma 105 Aldrich, M0). The supernatants obtained by centrifugation of homogenates (11, $000 \mathrm{~g}$ at $4^{\circ} \mathrm{C}$ for $30 \mathrm{~min}$ ) were collected and protein concentrations were determined using the micro BCA protein assay with a Multiscan EX (Thermo LabSystems) and Accent Software 2.6.

\section{Western Blotting analysis}

Proteins preparation homogenates were mixed with $5 x$ protein sample buffer $(62 \mathrm{mM}$ Tris- $\mathrm{HCl} \mathrm{pH} 6.8$, $2 \%$ SDS, $10 \%$ glycerol, $5 \%$ ß-mercaptoethanol, and $0.05 \%$ bromophenol blue as the tracking dye), and heated at $95^{\circ} \mathrm{C}$ for 5 minutes. Samples were then separated on 4-20\% SDS-PAGE gels and transferred into $0.22 \mu \mathrm{m}$ nitrocellulose membranes (Bio-Rad) using a Mini Trans-Blot Cell (Bio-Rad). Membranes were blocked in $5 \%$ non-fat dry milk in PBS $0.1 \%$ Tween and incubated overnight at $4{ }^{\circ} \mathrm{C}$ with anti-TRPC6 antibody (Alomone labs ACC-017, dilution 1:200). This was followed by a 1 hour incubation with a matched horseradish peroxidase labeled secondary antibody (Jackson, 1:3000). Immunoreactivity was detected using chemiluminescence detection kit reagents and a Chimidoc Station (Bio-Rad). To standardize for sample loading, the blots were subsequently stripped using a commercially available kit following the manufacturer's instructions (Reblot; Millipore), and reprobed with an anti- $\beta$-actin antibody (Sigma-Aldrich A5060, 1/1000) as an internal control for protein loading.

\section{Histological analysis of placentas}

The analysis of placental histology was done as described previously [18] [19]. Briefly, fourmicrometer-thick sections were cut from paraffin-embedded placenta blocks, mounted on silanized slides, deparaffinized, and rehydrated. The general morphology of the placenta with its 3 typical layers (decidua, junctional zone, and labyrinth) was analyzed on selected tissue sections after staining with hematoxylin and eosin. The thickness of each layer was measured using ImageJ (Image J software, 1.46r).

\section{Statistical analysis}

Differences between the 3 groups of mice were tested using one-way analysis of variance (ANOVA) followed by Bonferroni's post hoc test. When comparing 2 sets of data, a Student's t-test was used. A $P$ value $<0.05$ was considered statistically significant.

\section{Results}

TRPC6 $\%$ mice exhibit a reduced litter size

To determine whether TRPC6 invalidation influences the pregnancy outcome, we systematically counted at the day of birth the number of pups of C57Bl6/J, B6129SF2/J and TRPC6 $\%$ mice having a 129Sv:C57BL/6J genetic background [15]. The average size of the litter was $7.3 \pm 0.7,7.8 \pm 0.6$ and $5.1 \pm 0.5$ for the three strains, respectively (Fig. $1 \mathrm{~A})$. This shows that TRPC6 deficient mice had significantly less pups compared to $\mathrm{C} 57 \mathrm{Bl} 6 / \mathrm{J}$ and B6129SF2/J mice.

\section{TRPC6\% mice and developmental placenta factors}

Changes in mRNA levels of 4 markers of placental development were assessed using RT-qPCR: the transcription factors Mash2 and $\mathrm{Gcm} 1$, the placental hormone proliferin [20, 21], and CD31 (also called PECAM1), an endothelial cell protein [22, 23]. When compared to $\mathrm{C} 57 \mathrm{Bl6} / \mathrm{J}$ and $\mathrm{B} 6129 \mathrm{SF} 2 / \mathrm{J}$ mice, $\mathrm{TRPC}^{-/}$placentas displayed significant elevated mRNA 
levels of $\mathrm{CD} 31$ and $\mathrm{Gcm} 1 \quad(\mathrm{p}<0.05$, one-way ANOVA) (Fig. 1B). CD31 is a marker of endothelial cell junctions [22, 23] whereas Gcm 1 controls labyrinth branching [24-27]. No changes were observed in the levels of Mash2 and proliferin transcripts ( $p>0.05$, one-way ANOVA). Taken together, the deletion of TRPC6 gene caused significant changes in the expression of key placenta developmental factors, suggesting that TRPC $^{-/}$placentas may have developed compensatory processes to overcome the absence of the cation channel.

\section{TRPC6\% placentas are structurally disorganized}

The structure of C57Bl6/J and TRPC6 $\%$ placentas was analyzed. In these experiments, the thickness of each of the three placental layers (decidua, junctional zone, and labyrinth) was measured. Fig. 2 shows representative parasagittal sections of paraffin-embedded placentas collected at embryonic day 13 (E13) from C57Bl6/J (Fig. 2A) and TRPC6\% mice (Fig. 2B). The absence of TRPC6 did not affect the decidua but caused a narrowing of the labyrinth and an enlargement of the junctional zone. This is summarized in Fig. 2C-E with bar graphs illustrating the structural changes observed. In conclusion, the loss of TRPC6 disturbed the structure of the placenta.

\section{Placental Zn enrichment in TRPC6\% deficient mice}

The total content of Fe (Fig. 3A) and Zn (Fig. 3B) in placentas (left panels) and brain (right panels) from TRPC6 $^{-/}$mice and age-matched C57Bl6/J and B6129SF2/J mice was quantified by ICP-AES. Tissues were collected at E13 and processed for analysis. The loss of TRPC6 did not alter the amount of Fe in placenta and brain (Fig. 3A). However, in placentas from TRPC6-deficient mice $\mathrm{Zn}$ levels were increased by $\sim 15 \%$ and $\sim 40 \%$ when compared to $\mathrm{C} 57 \mathrm{Bl} / \mathrm{J}$ and B6129SF2/J mice, respectively ( $p<0.05$, one-way ANOVA followed by Bonferroni's test) (Fig. 3B). When similar measurements were conducted on brains, comparable amounts of

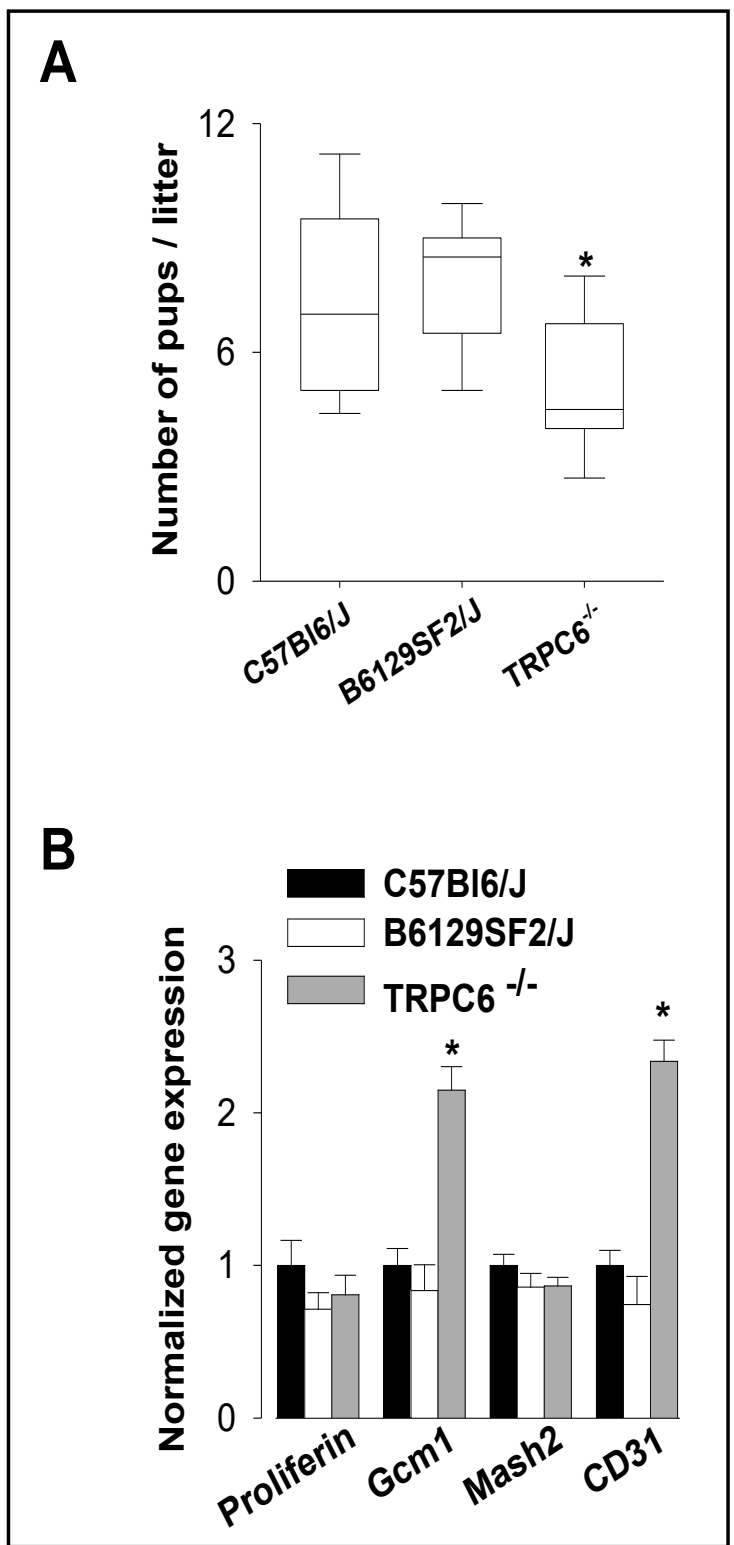

Fig. 1. Effect of the absence of TRPC6 on litter size and placental markers. A: The number of pups was systematically counted at post-natal day 1 (first day of birth) in C57Bl6/J, B6129SF2/J and TRPC6\% mice (with $\mathrm{n}=13,10$ and 16 gestations, respectively). ${ }^{*} \mathrm{p}<0.05$ (one-way ANOVA followed by Bonferroni's test) vs C57Bl6/J and vs B6129SF2/J mice. B: The relative abundance of genes involved in placenta development and functions (proliferin, Gcm1, Mash2, CD31) was analyzed by real-time RT-qPCR in the 3 groups of mice (C57Bl6/J, B6129SF2/J and TRPC6 ${ }^{-/}$). Data are normalized to $\mathrm{C} 57 \mathrm{Bl6} / \mathrm{J}$ mice with $\mathrm{n}=4$ per group. Mean \pm SEM with ${ }^{*} \mathrm{p}<0.05$ (one-way ANOVA followed by Bonferroni's test) vs C57Bl6/J and vs B6129SF2/J mice. 


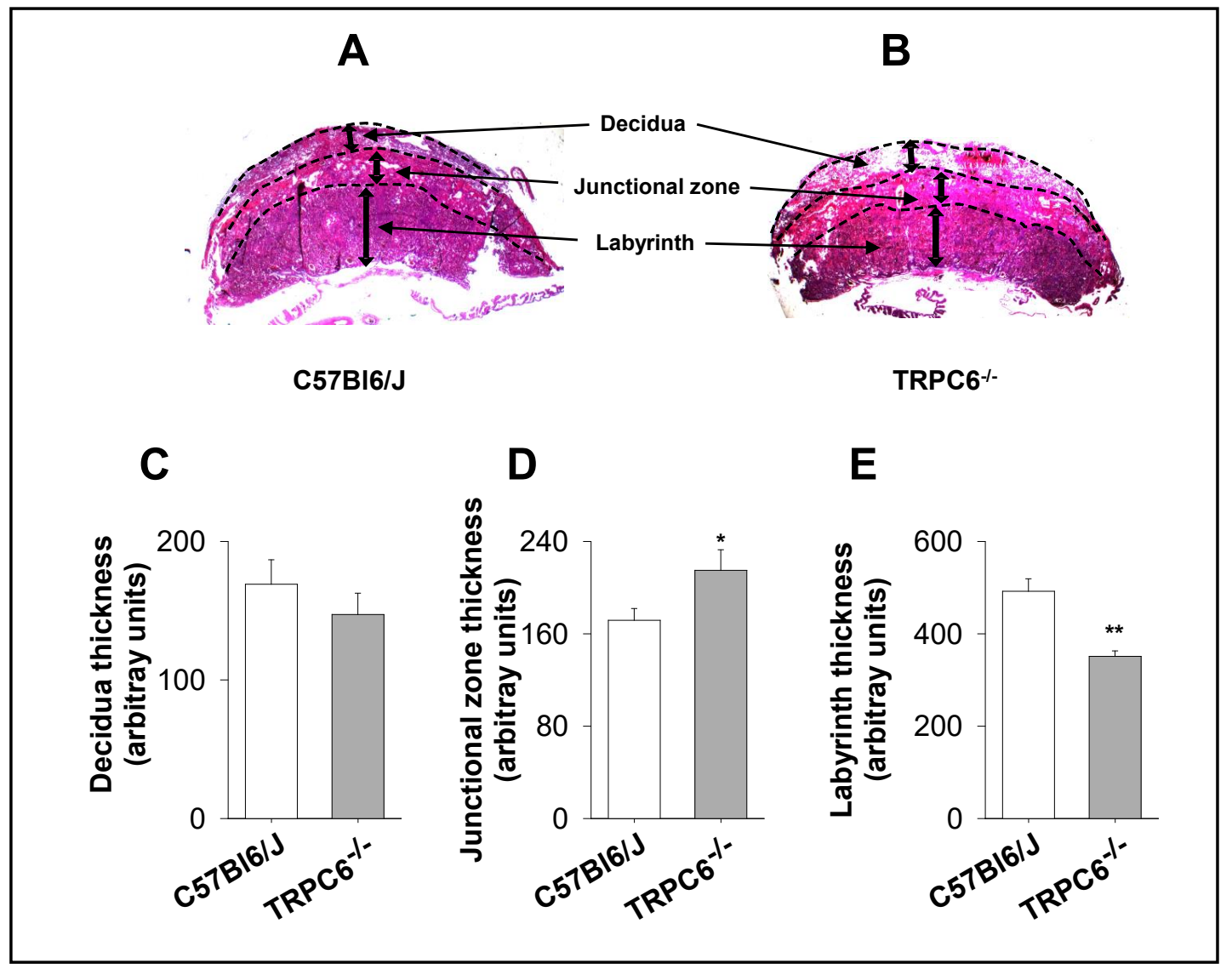

Fig. 2. Analysis of the placental layers. Panels A-B shows parasagittal sections of placentas from C57Bl6/J (A) and TRPC6 $\%$ mice (B) collected at E13 to identify the placental layers (decidua, junctional zone, and labyrinth). Panels C-E are bar graphs comparing the thickness of each placental layer in C57Bl6/J and TRPC6 $\%$ mice. Two parasagittal sections per placenta were analyzed with at least three placentas collected from three different mice. Mean $\pm \operatorname{SEM}\left({ }^{*} \mathrm{p}<0.05{ }^{* *} \mathrm{p}<0.01\right.$ Student's $\mathrm{t}$ test).

Zn were found in the 3 groups of mice (C57Bl6/J, B6129SF2/J and TRPC6 ${ }^{-/}$) (Fig. 3B). This showed that the deletion of TRPC6 was associated with a Zn enrichment in the placenta but not in the embryonic brain, indicating the existence of tissue specific alterations of the homeostasis of cations in TRPC6 ${ }^{-/}$mice.

\section{Zn and Fe contents in organs from neonatal mice}

Next, the content of Fe (Fig. 4A) and Zn (Fig. 4B) was measured in liver, kidney, lung and brain. Organs were collected on postnatal day 1 (PN1) (the day of birth) mice of both sex (C57Bl6/J, B6129SF2/J and TRPC6 ${ }^{-/}$). C57Bl6/J and B6129SF2/J pups exhibited distinct basal levels of Fe and $\mathrm{Zn}$ in liver. For instance, livers from B6129SF2/J pups had a lower Fe content when compared to livers from C57Bl6/J pups. High amounts of Zn were however found in liver and kidney from TRPC6\% pups when compared to C57Bl6/J. The liver of TRPC6-deficient pups contained less Fe than C57Bl6/J mice but the values were comparable to those found in B6129SF2/J pups (Fig. 4A). A clear Zn enrichment was however noted in TRPC6 $\%$ liver and kidney (Fig. 4B). For instance, the liver content of $\mathrm{Zn}$ was increased by $\sim 105 \%$ in TRPC $6^{-/}$mice when compared to $\mathrm{C} 57 \mathrm{Bl} / \mathrm{J}$ mice. A strong $\mathrm{Zn}$ enrichment was also noted in the kidney $(+\sim 160 \%$ and $+\sim 40 \%$, when compared to C57Bl6/J and B6129SF2/J mice, respectively). The absence of TRPC6 did not influence the amounts of Fe or Zn in the brain, indicating that the mineral status of this organ was uninfluenced by the deletion of TRPC6, at least in 1-day old pups. For the lung, a Zn and Fe overload may develop although 
the differences were not statistically different.

\section{Zn and Fe contents in organs from} adult mice

In this set of experiments, organs were collected from age-matched (3-6 months) adult females C57Bl6/J, B6129SF2/J and TRPC6 $^{-1}$ mice. The loss of TRPC6 was associated with higher levels of $\mathrm{Fe}$ in adult TRPC6 ${ }^{-/}$ brain. The amount of this metal was increased by $\sim 40 \%$ when compared to $\mathrm{C} 57 \mathrm{Bl6} / \mathrm{J}$ and $\mathrm{B} 6129 \mathrm{SF} 2 / \mathrm{J}$ mice $(p<0.5$, one-way ANOVA followed by Bonferroni's test) (Fig. 5A). A Fe overload was also present in the adult TRPC6 $^{-1}$ liver when compared to C57Bl6/J mice $(+\sim 56 \%$, $\mathrm{p}<0.05$, oneway ANOVA followed by Bonferroni's test) (Fig. 5A). However, comparable amounts of $\mathrm{Zn}$ were measured in organs from adult mice (Fig. 5B) ( $p>0.05$, one-way ANOVA followed by Bonferroni's test). In conclusion, the analysis of the Fe and $\mathrm{Zn}$ contents showed that the deletion of TRPC6 was accompanied by increased levels of $\mathrm{Zn}$ in placenta, liver and kidney at the embryonic and post-natal stages but not at adulthood. A Fe overload was however noted in brain and liver from adult TRPC $\%$ mice. On the other hand, the $\mathrm{Zn}$ and $\mathrm{Fe}$ status of the lung was unaffected by the deletion of TRPC6. These data indicate that TRPC6 deficient mice developed organ and age-dependent perturbations in their $\mathrm{Zn}$ and Fe status.

\section{TRPC6 expression in preeclampsia}

The experiments reported above showed that TRPC6 invalidation caused a marked placental Zn enrichment (+ 15-40\%), upregulated gene expression of CD31 and Gcm1, altered the placental histology and diminished the litter size. These data point to a role for TRPC6 in placental functions and pregnancy outcome. To further document the pathophysiological relevance of this channel, we investigated TRPC6 protein levels in preeclampsia (PE), a common human gestational pathology. Fig. 6A shows representative Western blots from control and PE placentas. There was a significant increase in TRPC6 protein levels in PE placentas ( $\mathrm{p}<0.05$, Student's t test) (Fig. 6B). This result shows that PE is associated with altered levels of TRPC6 proteins, a finding that further highlights the pathophysiological relevance of TRPC6 in placental functions and pregnancy. 
Fig. 4. Total $\mathrm{Fe}$ and $\mathrm{Zn}$ contents in liver, kidney, lung and brain from neonatal mice. The total amount of $\mathrm{Fe}$ and $\mathrm{Zn}$ was measured in liver $(n=6-8)$, kidney $(n=6-$ $8)$, lung $(n=6-8)$ and brain $(n=9-22)$ from C57Bl6/J, B6129SF2/J and TRPC $^{-} \%$ mice of both sex. Organs were collected on pups the day of birth. Panels $A$ and $B$ show the amount of $\mathrm{Fe}$ and $\mathrm{Zn}$, respectively, in $\mathrm{ng} / \mathrm{mg}$ of dried tissue with * $\mathrm{p}<0.05$ with one-way ANOVA followed by Bonferroni's test.

Fig. 5. Total amount of $\mathrm{Fe}$ and $\mathrm{Zn}$ in liver, kidney, lung and brain from adult mice. The graph shows the total amount of $\mathrm{Fe}$ (A) and $\mathrm{Zn} \mathrm{(B)} \mathrm{in} 4$ organs (liver, kidney, lung, brain) obtained from adult (3-6 months) females mice (C57Bl6/J, B6129SF2/J and TRPC6 $^{\%}$ ). Significant differences in Fe levels were found only in liver and brain. $* \mathrm{p}<0.05$ with one-way ANOVA followed by Bonferroni's test, with $\mathrm{n}=5$ organs per group.

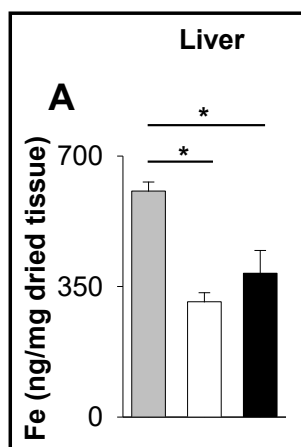

Kidney

Lung

Brain
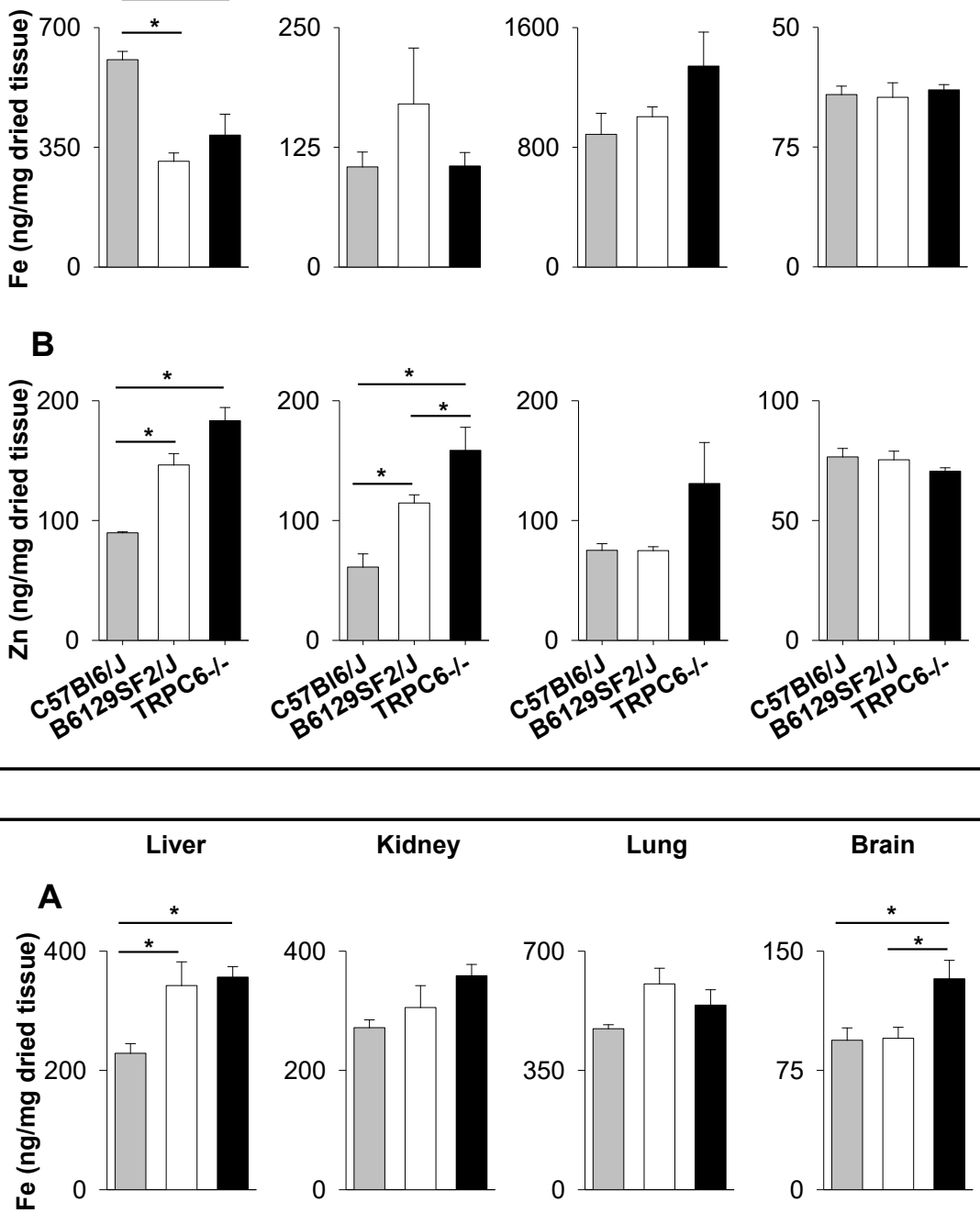

Kidney

Lung

Brain
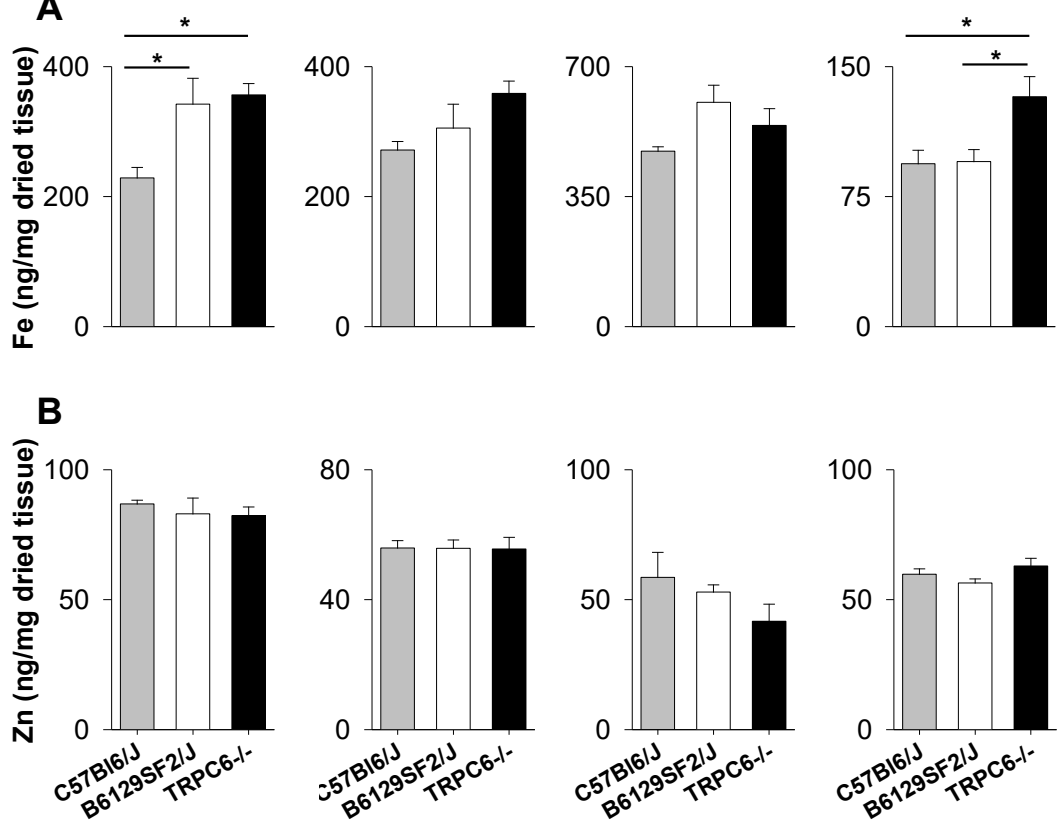
Fig. 6. TRPC6 proteins levels in human preeclampic placentas. The figure shows Western blots analysis (A) and quantification (B) of TRPC6 protein levels in control $(n=4)$ and preeclampic $(n=5)$ human placentas. Results were normalized to $\beta$-actin. * $\mathrm{p}<0.01$ Student's t-test.

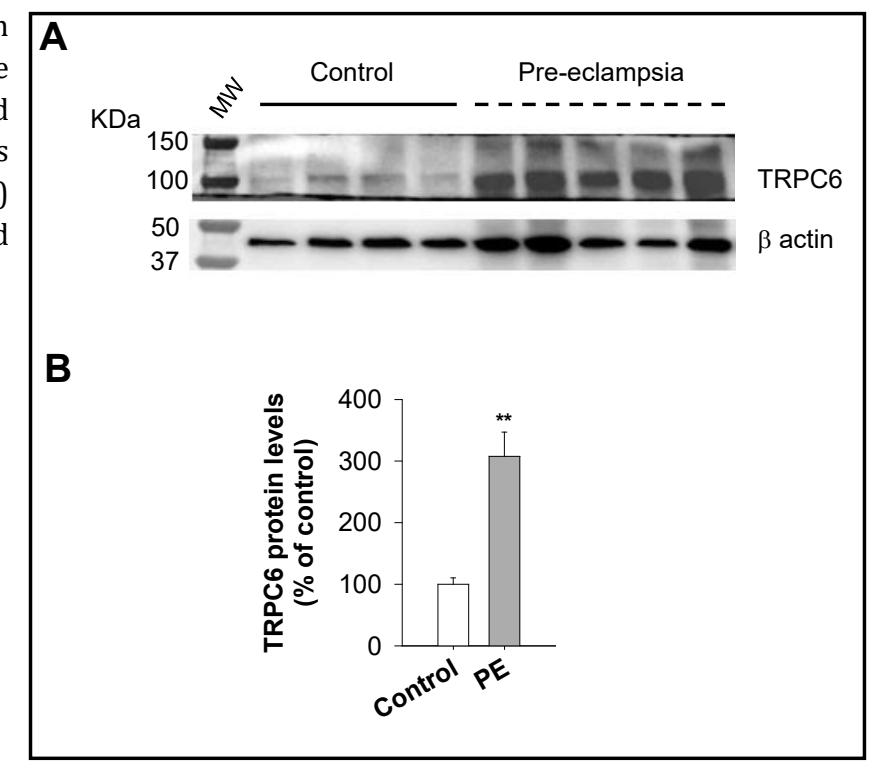

\section{Discussion}

The placenta is a unique organ that plays key roles in the transport of minerals, oxygen and nutrients. Various pathophysiological conditions can alter its functions and therefore influence the success of pregnancy. Human and murine placentas express high amounts of TRPC6 [2,11-13] but the physiological contribution of this channel to placental functions is currently unknown. To address that question we used a TRPC6-deficient mice strain [15]. TRPC6 $^{\%}$ females delivered less pups when compared to C57BL6/J and B6129SF2/J females mice. For instance, C57Bl6/J mice had an average of 6-7 pups per litter (see Mouse Phenome Database, http://www.jax.org/phenome) whereas TRPC6 ${ }^{-/}$mothers had 4-5. The number of pups at birth is an important physiological parameter with functional implications on various aspects of offspring development [28] such as age at sexual maturity and reproductive behaviors [29].

The deregulated pregnancy outcome was accompanied by histological modifications of the placenta. The thickness of the junctional zone was augmented, suggesting placental hypoxic conditions [30]. On the other hand, the surface of the labyrinth was significantly diminished, indicating a deregulation in placental branching with potential effects on fetomaternal exchanges [31]. In addition to these placental alterations, the loss of TRPC6 influenced the abundance of two key placental transcripts: CD31 (PECAM1) and Gcm1. CD31 is a transmembrane protein expressed by leukocytes and endothelial cells, being particularly enriched at endothelial cell intercellular junctions [22]. It is involved in various pathophysiological processes such as leukocyte migration through endothelial cell junctions, angiogenesis, mechano-transduction and vascular permeability. Importantly, CD31 co-localizes with TRPC6 at endothelial cell junctions. Furthermore, calcium imaging experiments reported that TRPC6 operates downstream to this endothelial cell adhesion molecule [32]. Our data show that the absence of TRPC6 alters CD31 mRNA expression and add further credit to the existence of a coupling between CD31 and TRPC6 in endothelial cells [32]. Gcm 1 is a trophoblast specific transcription factor [24, 25]. By promoting the expression of its target genes syncytin-1 and the placenta growth factor (PlGF), Gcm1 is a key regulator of placental development required for chorioallantoic branching and syncytiotrophoblast formation [33]. Similar to CD31, Gcm1 mRNA levels were significantly increased in TRPC6 ${ }^{-/}$placentas. It is interesting to note that Zn deficiency diminishes Gcm1 expression [34] whereas an excess of $\mathrm{Zn}$ is associated with high levels of $\mathrm{Gcm} 1$ transcripts (present study). 


\section{Cellular Physiology Cell Physiol Biochem 2019;52:455-467 \\ \begin{tabular}{ll|l} 
and Biochemistry $10.33594 / 000000033$ & P 2019 The Author(s). Published by \\
Published online: 15 March 2019 & Cell Physiol Biochem Press GmbH\&Co. KG
\end{tabular} \\ Hasna et al.: TRPC6 in Placenta and Mineral Homeostasis}

The $\mathrm{Zn}$ and Fe content of organs was quantified by atomic emission spectroscopy and the main findings are summarized in Table 3. During pregnancy, a significant $\mathrm{Zn}$ increase was noted in TRPC $^{\%}$ placentas. But this Zn overload had no effect on the brain $\mathrm{Fe}$ and $\mathrm{Zn}$ status of the fetuses. At birth liver and kidney from TRPC6 $^{-}$pups (post-natal day 1) displayed elevated $\mathrm{Zn}$ levels. Compared to $\mathrm{C} 57 \mathrm{Bl} 6 / \mathrm{J}$ mice, TRPC6 deficient pups had a reduced amount of $\mathrm{Fe}$ in liver but they were not different from B6129SF2/J mice. This indicates that the genetic background could influence metal homeostasis. On the other hand, at birth, brain and lung were uninfluenced by the loss of TRPC6. Interestingly, a dysregulation of the $\mathrm{Zn}$ status was observed postnatally (liver, kidney) but not at adulthood, showing that the deletion of TRPC6 caused a transient perturbation of Zn homeostasis. This suggests that organs develop compensatory mechanisms. Adult TRPC6 deficient mice manifest a Fe overload phenotype in brain $(+\sim 40 \%)$ and liver (+ 50\%) as compared with C57Bl6/J mice.

Overall, the present study shows that the loss of TRPC6 is associated with organ and age-dependent perturbations in $\mathrm{Zn}$ and Fe status. Interestingly, the lung was unaffected by the deletion of TRPC6, suggesting that the channel does not play any role in the mineral status of this organ. Alternatively, TRPC $6^{*}$ lung may have developed efficient compensatory mechanisms.

Another key finding of this study is the presence of elevated amounts of TRPC6 proteins in preeclampic placentas. Several pregnancy pathologies like PE, preterm delivery, or intrauterine growth restriction are associated with disrupted mineral homeostasis $[35,36]$ and altered expression of some cation channels such as TRPM6 and TRPM7 [37]. Collectively, the data collected on murine and human tissues indicate that TRPC6 is critical for a normal placental development.

Studies aiming at understanding the contribution of TRPC6 in Zn homeostasis have led to conflicting results. The over expression of TRPC6 in HEK cells as well as the deletion of TRPC6 gene are 2 maneuvers elevating intracellular Zn levels [7, 8] (present report). The different experimental approaches and biological models used may explain these counterintuitive findings. The impact of the deletion of TRPC6 on cation homeostasis in a particular organ or cell type may depend on intrinsic TRPC6 protein levels and the abundance of other putative redundant transport systems. The deletion of TRPC6 may have influenced various transport and signaling systems. For instance, a whole-genome study showed that the knockdown of the metal cation transporter gene ZIP8 (Slc39a8) results in an up- and down-regulation of numerous other transport genes [38]. Furthermore, a complete transcriptomic analysis by RNA-seq concluded that the loss of the TRPC 3 gene also up- and down-regulates $>150$ genes involved in various biological processes such as cell motility, lipid kinase activity, inositol lipid and Ca signaling [39].
Table 3. Summary of the measurement of the metal content. of TRPC6 on the Fe and Zn status of organs from TRPC6 I- mice collected at different ages (E13: at embryonic day females). $\lambda$ and $\searrow$ indicate respectively an augmentation C57Bl6J/J mice; n.d. : content not determined. = content not

\begin{tabular}{lccc}
\hline & \multicolumn{3}{c}{ Age } \\
Tissue & E13 & PN & Adult \\
\hline Brain & $=$ & $=$ & $\mathrm{Fe} \lambda$ \\
Liver & n.d. & $\mathrm{Fe} \searrow$ & $\mathrm{Fe} \lambda$ \\
& & $\mathrm{Zn} \lambda$ & $=$ \\
Lung & n.d. & $=$ & $=$ \\
Kidney & n.d. & $\mathrm{Zn} \lambda$ & $=$ \\
Placenta & Zn $\nearrow$ & n.d. & n.d. \\
\hline
\end{tabular}
significantly different 


\section{Cellular Physiology Cell Physiol Biochem 2019;52:455-467

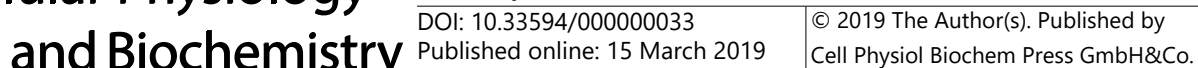 \\ Hasna et al.: TRPC6 in Placenta and Mineral Homeostasis}

\section{Conclusion}

A mouse strain deficient in TRPC6 shows altered amounts of Zn (or Fe) in the placenta, liver, kidney and brain but not in the lung. These metal dysregulations (excess or deficit) developed in an age- and tissue-dependent manner. Our data add insight into the growing body of evidence indicating that TRPC6 could play a role in mineral homeostasis. In addition, the loss of TRPC6 induced a transcriptional regulation of CD31 and Gcm1, two genes involved in placental development and function. It also reduced litter sizes associated with placental histological abnormalities. Moreover, preeclampic placentas have elevated amounts of TRPC6 proteins. So far, the roles of TRPC6 in placenta are largely unknown but our data point to critical pathophysiological functions.

\section{Acknowledgements}

We also wish to acknowledge support from the Centre National de la Recherche Scientifique (CNRS), Commissariat à l'Energie Atomique et aux Energies Alternatives (CEA), l'Institut National de la Santé et de la Recherche Médicale (INSERM) and the Université de Grenoble Alpes (UGA). Subjects have given their written informed consent. Animal experiments were conducted in compliance with the French legislation and the European Community Council Directive of 24 November 1986 (86/609/EEC). The experimental protocol was approved by the ethical committee of the CEA's Life Sciences Division (CETEA). The study was supported by grants from l'Agence Nationale de la Recherche (ANR-13NEUR-0003-02 and ANR-16-CE29-0024 to AB) and the Fondation pour la recherche Médicale (FRM) (SPF20150934074 to RAN). The funders had no role in study design, data collection and analysis, decision to publish, or preparation of the manuscript.

\section{Disclosure Statement}

The authors have no conflicts of interest to declare.

\section{References}

1 Dietrich A, Gudermann T: TRPC6, in Flockerzi V, Nilius B (eds): Transient Receptor Potential (TRP) Channels. Handbook of Experimantal Pharmacology, vol 179, Springer, Berlin, Heidelberg, 2007, pp 125141.

2 Hofmann T, Obukhov AG, Schaefer M, Harteneck C, Gudermann T, Schultz G: Direct activation of human TRPC6 and TRPC3 channels by diacylglycerol. Nature 1999;397:259-263.

3 Leuner K, Kazanski V, Muller M, Essin K, Henke B, Gollasch M, Harteneck C, Muller WE: Hyperforin--a key constituent of St. John's wort specifically activates TRPC6 channels. Faseb J 2007;21:4101-4111.

4 Dryer SE, Kim EY: Permeation and Rectification in Canonical Transient Receptor Potential-6 (TRPC6) Channels. Front Physiol 2018;9:1055.

5 Mwanjewe J, Grover AK: Role of transient receptor potential canonical 6 (TRPC6) in non-transferrin-bound iron uptake in neuronal phenotype PC12 cells. Biochem J 2004;378:975-982.

6 Knutson MD: Non-transferrin-bound iron transporters. Free Radic Biol Med 2019;133:101-111.

- 7 Gibon J, Tu P, Bohic S, Richaud P, Arnaud J, Zhu M, Boulay G, Bouron A: The over-expression of TRPC6 channels in HEK-293 cells favours the intracellular accumulation of zinc. Biochim Biophys Acta 2011;1808:2807-2818.

8 Chevallet M, Jarvis L, Harel A, Luche S, Degot S, Chapuis V, Boulay G, Rabilloud T, Bouron A: Functional consequences of the over-expression of TRPC6 channels in HEK cells: impact on the homeostasis of zinc. Metallomics 2014;6:1269-1276. 


\section{Cellular Physiology Cell Physiol Biochem 2019;52:455-467 \begin{tabular}{c|c|c|c|}
\hline DOI: 10.33594/000000033 & (O) 2019 The Author(s). Published by \\
\hline
\end{tabular} and Biochemistry Published online: 15 March 2019 Cell Physiol Biochem Press GmbH\&Co. KG \\ Hasna et al.: TRPC6 in Placenta and Mineral Homeostasis}

- 9 Boulay G, Zhu X, Peyton M, Jiang M, Hurst R, Stefani E, Birnbaumer L: Cloning and expression of a novel mammalian homolog of Drosophila transient receptor potential (Trp) involved in calcium entry secondary to activation of receptors coupled by the Gq class of G protein. J Biol Chem 1997;272:29672-29680.

- 10 Bouron A, Chauvet S, Dryer S, Rosado JA: Second Messenger-Operated Calcium Entry Through TRPC6. Adv Exp Med Biol 2016;898:201-249.

- 11 Riccio A, Medhurst AD, Mattei C, Kelsell RE, Calver AR, Randall AD, Benham CD, Pangalos MN: mRNA distribution analysis of human TRPC family in CNS and peripheral tissues. Brain Res Mol Brain Res 2002;109:95-104.

12 Clarson LH, Roberts VH, Hamark B, Elliott AC, Powell T: Store-operated Ca2+ entry in first trimester and term human placenta. J Physiol 2003;550:515-528.

13 Fagerberg L, Hallstrom BM, Oksvold P, Kampf C, Djureinovic D, Odeberg J, Habuka M, Tahmasebpoor S, Danielsson A, Edlund K, Asplund A, Sjostedt E, Lundberg E, Szigyarto CA, Skogs M, Takanen JO, Berling $\mathrm{H}$, Tegel H, Mulder J, Nilsson P, et al.: Analysis of the human tissue-specific expression by genome-wide integration of transcriptomics and antibody-based proteomics. Mol Cell Proteomics 2014;13:397-406.

- 14 Sibai B, Dekker G, Kupferminc M: Pre-eclampsia. Lancet 2005;365:785-799.

- 15 Dietrich A, Mederos YSM, Gollasch M, Gross V, Storch U, Dubrovska G, Obst M, Yildirim E, Salanova B, Kalwa H, Essin K, Pinkenburg O, Luft FC, Gudermann T, Birnbaumer L: Increased vascular smooth muscle contractility in TRPC6-/- mice. Mol Cell Biol 2005;25:6980-6989.

- 16 Alfaidy N, Gupta S, DeMarco C, Caniggia I, Challis JR: Oxygen regulation of placental 11 beta-hydroxysteroid dehydrogenase 2: physiological and pathological implications. J Clin Endocrinol Metab 2002;87:47974805.

17 Chesley LC: Diagnosis of preeclampsia. Obstet Gynecol 1985;65:423-425.

- 18 Holloway AC, Salomon A, Soares MJ, Garnier V, Raha S, Sergent F, Nicholson CJ, Feige JJ, Benharouga M, Alfaidy N: Characterization of the adverse effects of nicotine on placental development: in vivo and in vitro studies. Am J Physiol Endocrinol Metab 2014;306:E443-E456.

19 Sergent F, Hoffmann P, Brouillet S, Garnier V, Salomon A, Murthi P, Benharouga M, Feige JJ, Alfaidy N: Sustained Endocrine Gland-Derived Vascular Endothelial Growth Factor Levels Beyond the First Trimester of Pregnancy Display Phenotypic and Functional Changes Associated With the Pathogenesis of PregnancyInduced Hypertension. Hypertension 2016;68:148-156.

20 Haig D: Placental growth hormone-related proteins and prolactin-related proteins. Placenta 2008;29:S3641.

21 Linzer DI, Fisher SJ: The placenta and the prolactin family of hormones: regulation of the physiology of pregnancy. Mol Endocrinol 1999;13:837-840.

22 Ebnet K: Junctional Adhesion Molecules (JAMs): Cell Adhesion Receptors With Pleiotropic Functions in Cell Physiology and Development. Physiol Rev 2017;97:1529-1554.

- 23 Lertkiatmongkol P, Liao D, Mei H, Hu Y, Newman PJ: Endothelial functions of platelet/endothelial cell adhesion molecule-1 (CD31). Curr Opin Hematol 2016;23:253-259.

24 Cross JC, Anson-Cartwright L, Scott IC: Transcription factors underlying the development and endocrine functions of the placenta. Recent Prog Horm Res 2002;57:221-234.

25 Rawn SM, Cross JC: The evolution, regulation, and function of placenta-specific genes. Annu Rev Cell Dev Biol 2008;24:159-181.

26 Guillemot F, Nagy A, Auerbach A, Rossant J, Joyner AL: Essential role of Mash-2 in extraembryonic development. Nature 1994;371:333-336.

27 Scott IC, Anson-Cartwright L, Riley P, Reda D, Cross JC: The HAND1 basic helix-loop-helix transcription factor regulates trophoblast differentiation via multiple mechanisms. Mol Cell Biol 2000;20:530-541.

28 Tanaka T: Effects of litter size on behavioral development in mice. Reprod Toxicol 1998;12:613-617.

- 29 Mendi M: The effects of litter size variation on mother-offspring relationships and behavioural and physical development in several mammalian species (principally rodents). J Zool Lond 1988;215:15-34.

30 Cowden Dahl KD, Fryer BH, Mack FA, Compernolle V, Maltepe E, Adelman DM, Carmeliet P, Simon MC: Hypoxia-inducible factors 1alpha and 2alpha regulate trophoblast differentiation. Mol Cell Biol 2005;25:10479-10491.

31 Rossant J, Cross JC: Placental development: lessons from mouse mutants. Nat Rev Genet 2001;2:538-548. 


\section{Cellular Physiology and Biochemistry}

Cell Physiol Biochem 2019;52:455-467

\begin{tabular}{l|l}
\hline DOI: 10.33594/000000033 & (c) 2019 The Author(s). Published by \\
\hline
\end{tabular}

Hasna et al.: TRPC6 in Placenta and Mineral Homeostasis

32 Weber EW, Han F, Tauseef M, Birnbaumer L, Mehta D, Muller WA: TRPC6 is the endothelial calcium channel that regulates leukocyte transendothelial migration during the inflammatory response. J Exp Med 2015;212:1883-1899.

33 Anson-Cartwright L, Dawson K, Holmyard D, Fisher SJ, Lazzarini RA, Cross JC: The glial cells missing-1 protein is essential for branching morphogenesis in the chorioallantoic placenta. Nat Genet 2000;25:311314.

34 Tian X, Anthony K, Neuberger T, Diaz FJ: Preconception zinc deficiency disrupts postimplantation fetal and placental development in mice. Biol Reprod 2014;90:83.

35 Alfaidy N, Chauvet S, Donadio-Andrei S, Salomon A, Saoudi Y, Richaud P, Aude-Garcia C, Hoffmann P, Andrieux A, Moulis JM, Feige JJ, Benharouga M: Prion protein expression and functional importance in developmental angiogenesis: role in oxidative stress and copper homeostasis. Antioxid Redox Signal 2013;18:400-411.

36 Ranjkesh F, Jaliseh HK, Abutorabi S: Monitoring the copper content of serum and urine in pregnancies complicated by preeclampsia. Biol Trace Elem Res 2011;144:58-62.

37 Yang H, Kim TH, Lee GS, Hong EJ, Jeung EB: Comparing the expression patterns of placental magnesium/ phosphorus-transporting channels between healthy and preeclamptic pregnancies. Mol Reprod Dev 2014;81:851-860.

38 Chen J, Galvez-Peralta M, Zhang X, Deng J, Liu Z, Nebert DW: In utero gene expression in the Slc39a8(neo/ neo) knockdown mouse. Sci Rep 2018;8:10703.

39 Kumarasamy S, Solanki S, Atolagbe OT, Joe B, Birnbaumer L, Vazquez G: Deep Transcriptomic Profiling of M1 Macrophages Lacking Trpc3. Sci Rep 2017;7:39867. 